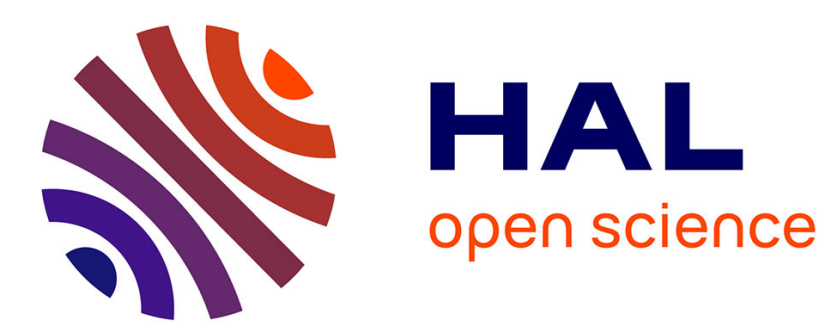

\title{
Traffic Grooming in Unidirectional WDM Ring Networks using Design Theory
}

\author{
Jean-Claude Bermond, David Coudert
}

\section{To cite this version:}

Jean-Claude Bermond, David Coudert. Traffic Grooming in Unidirectional WDM Ring Networks using Design Theory. IEEE International Conference on Communications (ICC '03)., May 2003, Anchorage, United States. pp.1402 - 1406, 10.1109/ICC.2003.1204621 . inria-00429174

\section{HAL Id: inria-00429174 \\ https://hal.inria.fr/inria-00429174}

Submitted on 1 Nov 2009

HAL is a multi-disciplinary open access archive for the deposit and dissemination of scientific research documents, whether they are published or not. The documents may come from teaching and research institutions in France or abroad, or from public or private research centers.
L'archive ouverte pluridisciplinaire HAL, est destinée au dépôt et à la diffusion de documents scientifiques de niveau recherche, publiés ou non, émanant des établissements d'enseignement et de recherche français ou étrangers, des laboratoires publics ou privés. 


\title{
Traffic Grooming in Unidirectional WDM Ring Networks using Design Theory
}

\author{
Jean-Claude Bermond, David Coudert, IEEE Member \\ MASCOTTE project, I3S-CNRS/InRIA/Université de Nice-Sophia Antipolis, \\ B.P. 93, F-06902 Sophia Antipolis Cedex, FranCE.
}

\begin{abstract}
We address the problem of traffic grooming in WDM rings with all-to-all uniform unitary traffic. We want to minimize the total number of SONET add-drop multiplexers (ADMs) required. We show that this problem corresponds to a partition of the edges of the complete graph into subgraphs, where each subgraph has at most $C$ edges (where $C$ is the grooming ratio) and where the total number of vertices has to be minimized. Using tools of graph and design theory, we optimally solve the problem for practical values and infinite congruence classes of values for a given $C$, and thus improve and unify all the preceding results. We disprove a conjecture of [7] saying that the minimum number of ADMs cannot be achieved with the minimum number of wavelengths, and also another conjecture of [17].

Keywords: Traffic grooming, graph, design theory, WDM rings.
\end{abstract}

\section{INTRODUCTION}

The WDM (Wavelength Division Multiplexing) has significantly increased the available capacity transmission of networks, therefore the bottleneck is now in the nodes (routers) where all the information has to be processed. In order to reduce the cost of the network, it becomes important to reduce the traffic processed at the node. Traffic grooming is the generic term for packing low rate signals into higher speed streams (see the surveys $[14,19,20]$ ). By using traffic grooming, one can bypass the electronics in the nodes for which there is no traffic sourced or destinated to it. Typically, in a WDM network, instead of having one SONET Add Drop Multiplexer (shortly ADM) on every wavelength at every node, it may be possible to have ADMs only for the wavelength used at that node (the other wavelengths being optically routed without electronic switching).

In the past many papers on WDM networks had for objective to minimize the transmission cost and in particular the number of wavelengths to be used $[1,11,12]$, recent research has focused on reducing the total number of ADMs used in the network, trying to minimize it.

It is known that even for the simpler network which is the unidirectional ring, the number of wavelengths and the number of ADMs cannot be simultaneously minimized (see [15], or [7] for uniform traffic). Furthermore, given a traffic matrix expressed in some units of a bandwidth (for example OC-3) where $r_{i, j}$ units have to be transmitted from $i$ to $j$, the solution will depend on the routing used and how connections are assigned to wavelengths. Hence, the general problem is very difficult.

Here, we consider the particular case of unidirectional rings (the routing is unique) with static uniform symmetric all-to-all traffic (that is $r_{i, j}=1$ for all couples $(i, j)$ ) and with no possible wavelength conversion.

In that case, for each pair $\{i, j\}$, we associate a circle (or circuit) which contains both the request from $i$ to $j$ and from $j$ to $i$. If each circle requires only $\frac{1}{C}$ of the bandwidth of a wavelength, we can "groom" $C$ circles on the same wavelength. $C$ is called the grooming ratio (or grooming factor). For example, if the request from $i$ to $j$ (and from $j$ to $i$ ) is one OC-12 and a wavelength can carry an OC-48, the grooming factor is 4 . Given the grooming ratio $C$ and the size $N$ of the ring, the objective is to minimize the total number of (SONET) ADMs used, denoted $A(C, N)$, and so reducing the network cost by eliminating as many ADMs as possible from the "no grooming case". For example, let $N=4$; we have 6 circles corresponding to the 6 pairs $\{0,1\},\{0,2\},\{0,3\},\{1,2\},\{1,3\},\{2,3\}$. If we don't use grooming, that is if we assign one wavelength per circle, we need 2 ADMs per circle, and thus a total of 12. Suppose now that $C=4$, that is we can groom 4 circles on one wavelength. One can groom on wavelength 1 the circles

Www: http://www-sop.inria.fr/mascotte

E-mail: \{Jean-Claude.Bermond,David.Coudert\}@sophia.inria.fr.

This work has been partially funded by European projects RTN ARACNE and FET CRESCCO. 
TABLE I

$A(C, N)$ FOR $N \leq 16$ AND $C=3,4,12,16,48,64$

\begin{tabular}{|l||c|c|c|c|c|c|c|c|c|c|c|c|c|c|}
\hline & $\mathrm{N}=3$ & $\mathrm{~N}=4$ & $\mathrm{~N}=5$ & $\mathrm{~N}=6$ & $\mathrm{~N}=7$ & $\mathrm{~N}=8$ & $\mathrm{~N}=9$ & $\mathrm{~N}=10$ & $\mathrm{~N}=11$ & $\mathrm{~N}=12$ & $\mathrm{~N}=13$ & $\mathrm{~N}=14$ & $\mathrm{~N}=15$ & $\mathrm{~N}=16$ \\
\hline \hline $\mathrm{C}=3[2]$ & 3 & 7 & 12 & 17 & 21 & 31 & 36 & 48 & 57 & 69 & 78 & 95 & 105 & 124 \\
\hline $\mathrm{C}=4$ & 3 & 7 & 10 & 15 & 21 & 28 & 36 & 45 & 55 & 66 & 78 & 91 & 105 & 120 \\
\hline $\mathrm{C}=12$ & 3 & 4 & 5 & 9 & 12 & 16 & 18 & 24 & 30 & 35 & 39 & 47 & $55-56$ & 60 \\
& & & & $9-9$ & $11-12$ & $15-16$ & $18-18$ & $23-24$ & $28-30$ & $33-36$ & $39-39$ & $46-49$ & $53-57$ & $60-64$ \\
\hline $\mathrm{C}=16$ & 3 & 4 & 5 & 6 & 11 & 14 & 18 & 20 & 26 & 32 & 36 & 41 & 45 & $53-54$ \\
& & & & & $10-11$ & $12-14$ & $16-18$ & $18-20$ & $23-26$ & $28-33$ & $33-37$ & $37-42$ & $42-46$ & $48-57$ \\
\hline $\mathrm{C}=48$ & 3 & 4 & 5 & 6 & 7 & 8 & 9 & 10 & 16 & 19 & 22 & 24 & 30 & 32 \\
& & & & & & & & & $15-16$ & $17-19$ & $19-22$ & $21-24$ & $26-31$ & $29-34$ \\
\hline $\mathrm{C}=64$ & 3 & 4 & 5 & 6 & 7 & 8 & 9 & 10 & 11 & 15 & 19 & 22 & 25 & 28 \\
& & & & & & & & & & $15-15$ & $18-19$ & $20-22$ & $22-25$ & $24-28$ \\
\hline
\end{tabular}

associated to $\{0,1\},\{1,2\},\{2,3\},\{3,0\}$ requiring 4 ADMs and on wavelength 2 the circles associated to $\{0,2\}$ and $\{1,3\}$ requiring 4 ADMs and so a total of 8 . A better way is to groom the circles associated to $\{0,1\},\{0,2\},\{0,3\}$ using 4 ADMs and those associated with $\{1,2\},\{1,3\},\{2,3\}$ using 3 ADMs and a total of 7 ADMs.

The case we consider has been considered by many authors [7,13,15-17,21,22, 24-26] and numerical results, heuristics and tables have been given (see for example that in [22]). It presents the advantage of concentrating on the grooming phase (excluding the routing). It can also be applied to groom components of more general connections than two opposite pairs into wavelengths or more general classes. These components are called circles $[7,26]$ or circuits [22] or primitive rings $[9,10]$.

Here we show that the problem of minimizing the number of ADMs for the unidirectional ring $C_{N}$ with a grooming factor $C$ can be expressed as follows: partition the edges of the complete graph on $N$ vertices $\left(K_{N}\right)$ into $W$ subgraphs $B_{\lambda}, \lambda=1,2, \ldots, W$, having $\left|E\left(B_{\lambda}\right)\right|$ edges and $\left|V\left(B_{\lambda}\right)\right|$ vertices with $\left|E\left(B_{\lambda}\right)\right| \leq C$ and where $\sum_{\lambda=1}^{W}\left|V\left(B_{\lambda}\right)\right|$ has to be minimized (the edges of $K_{N}$ correspond to the circles, the subgraphs $B_{\lambda}$ correspond to the wavelengths and a node of $B_{\lambda}$ corresponds to an ADM).

We show the importance of choosing graphs $B_{\lambda}$ in the partition with the best ratio $\frac{\left|E\left(B_{\lambda}\right)\right|}{\left|V\left(B_{\lambda}\right)\right|}$. Indeed, if we denote by $\rho_{\max }(C)$ the maximum ratio among all graphs with at most $C$ edges, we have the following lower bound on the minimum number $A(C, N)$ of ADMs: $A(C, N) \geq \frac{N(N-1)}{2 \rho_{\max }(C)}$.

For some values of $C$ the best ratio is obtained for subgraphs with $C$ edges, but it is not the case for other values of $C$, namely if $\frac{k(k-1)}{2}<C<\frac{(k+1)(k-1)}{2}$, where the best ratio is attained for the complete graph $K_{k}$. For these values of $C$, design theory enables us to give counterexamples to the conjecture of [7] saying that "the minimum number of ADMs, $A(C, N)$, for unidirectional rings with uniform unitary traffic is obtained using the minimum number of wavelengths" is false, the smallest case being $C=7$. That also give counterexamples for $C=16$ and to the conjecture of [17].

We show furthermore that the techniques of design theory can be used to obtain optimal or quasi-optimal results that improve all the preceding results of the literature and unify them. Thus, it is possible to use the vast effort and the numerical results obtained in the last century in design theory [8], without reinventing them. Note that design theory was also used in $[9,10]$ for $C=8$.

Among the results we have obtained on $A(C, N)$ are the following.

- Given $C$, when $N$ is large enough, $A(C, N)=\frac{N(N-1)}{2 \rho_{\max }(C)}$.

- Exact results for $C=3, C=4$ (already obtained in [17]) and for various congruence classes, for other values of $C$.

- Table I with the same entries as in [22] but with optimal values. Table I show the values of $A(C, N)$ for $N \leq 16$ and $C=3,4,12,16,48,64$. We have indicated in the line below the best known values from Table III of [22] in the form $l(C, N)-u(C, N)$ where $l(C, N)$ is the lower bound of [22] and $u(C, N)$ the best result obtained by heuristics. 


\section{NOTATION AND REFORMULATION OF THE PROBLEM}

We precise here our notations and show how the problem can be formulated in terms of graph partitioning. Although we restrict ourselves to the case of unidirectional rings with uniform static unit traffic, the ideas can be applied to other situations.

- $N$ will denote the number of nodes of the unidirectional ring $\vec{C}_{N}$

- For the unidirectional ring with symmetric traffic, $C_{\{i, j\}}$ will denote a circle associated to the pair $\{i, j\}$, that is containing both an unitary request from $i$ to $j$ and from $j$ to $i$. So $C_{\{i, j\}}$ uses all the $\operatorname{arcs}$ of $\vec{C}_{N}$.

- $R$ the total number of circles. In the case of unidirectional rings, with uniform unitary traffic, each pair $\{i, j\}$ is associated to a unique circle $C_{\{i, j\}}$ and thus $R=\frac{N(N-1)}{2}$.

- $C$ the grooming ratio (or grooming factor). In the example of [7], $C$ indicates the number of circles a wavelength can contain. Similarly, $\frac{1}{C}$ indicates the part of the bandwidth of a wavelength that can be used by a circle. For example, if a wavelength is running at the line rate of OC- $N$, it can carry $C=\frac{N}{M}$ low speed OC- $M$. Typical values of $C$ are $C=3,4,8,12,16,48,64$.

- Let $K_{N}$ be the complete graph on $N$ vertices where there is an edge $\{i, j\}$ for each pair of vertices $\{i, j\}$; let $C_{N}$ be the undirected cycle with $N$ nodes.

- $B_{\lambda}$ will denote a subgraph of $K_{N} . V\left(B_{\lambda}\right)$ (resp $E\left(B_{\lambda}\right)$ ) denote its vertex (resp edge) set. In the example of the introduction, $B_{\lambda}$ corresponds to a wavelength ; an edge $\{i, j\}$ of $B_{\lambda}$ correspond to a circle $C_{\{i, j\}}$. So a subgraph can be viewed as the set of circles packed in the wavelength. The grooming factor implies that $\left|E\left(B_{\lambda}\right)\right| \leq C$. $V\left(B_{\lambda}\right)$ corresponds to the number of (SONET) ADMs used in the wavelength $\lambda$; indeed we have to use an ADM in all the vertices appearing in a circle $C_{\{i, j\}}$ packed in the wavelength $\lambda$.

So, the original problem of minimizing the total number $A(C, N)$ of ADMs in a grooming with grooming ratio $C$, in the unidirectional ring $\vec{C}_{N}$ with unitary static uniform traffic, can be stated as follows.

Problem II.1-ADM:

Inputs : a number of nodes $N$ and a grooming ratio

C

Output: a partition of the edges of $K_{N}$ into subgraphs $B_{\lambda}, \lambda=1, \ldots, W$, such that $\left|E_{\lambda}\right| \leq C$

Objective :minimize $\sum_{1 \leq \lambda \leq W}\left|V_{\lambda}\right|$

Remark: As we said in the introduction, most interest has focused on a different objective function which was to minimize the number $W$ of subgraphs (wavelengths) of the partition. This is in this context an easy problem as $W_{\min }=\left\lceil\frac{R}{C}\right\rceil=\left\lceil\frac{N(N-1)}{2 C}\right\rceil$.

\section{LOWER BOUND}

Let $\rho\left(B_{\lambda}\right)$ denote the ratio of a subgraph $B_{\lambda}, \rho\left(B_{\lambda}\right)=\frac{\left|E\left(B_{\lambda}\right)\right|}{\left|V\left(B_{\lambda}\right)\right|}$, and $\rho(m)$ the maximum ratio of a subgraph with $m$ edges. Let $\rho_{\max }(C)$ denote the maximum ratio of subgraphs with $m \leq C$ edges. We have $\rho_{\max }(C)=$ $\max \left\{\rho\left(B_{\lambda}\right)|| E\left(B_{\lambda}\right) \mid \leq C\right\}=\max _{m \leq C} \rho(m)$.

Theorem III.1: Any grooming of $R$ circles with a grooming factor $C$ needs at least $\frac{R}{\rho_{\max }(C)}$ ADMs.

Proof: We have $R=\sum_{\lambda=1}^{W}\left|E\left(B_{\lambda}\right)\right| \leq \rho_{\max }(C) \sum_{\lambda=1}^{W}\left|V\left(B_{\lambda}\right)\right|$.

In particular, we get the following lower bound

Theorem III.2-Lower Bound: $A(C, N) \geq \frac{N(N-1)}{2 \rho_{\max }(C)}$.

We will now compute $\rho_{\max }(C)$. Let $\varphi(m)=\min \left\{k \mid \frac{k(k-1)}{2} \geq m\right\}$, that is $\varphi(m)=\left\lceil\frac{1+\sqrt{1+8 m}}{2}\right\rceil$ and note that any subgraph with $m$ edges has at least $\varphi(m)$ vertices.

Proposition III.3: If $\frac{k(k-1)}{2} \leq C \leq \frac{(k+1)(k-1)}{2}$, then $\rho_{\max }(C)=\frac{k-1}{2}$ and the value is attained for $K_{k}$.

If $\frac{(k+1)(k-1)}{2} \leq C \leq \frac{(k+1) k}{2}$, then $\rho_{\max }(C)=\frac{C}{k+1}$ and the value is attained for any graph with $C$ edges and $k+1$ vertices. 
TABLE II

VALUES OF $\rho_{\max }(C)$ FOR SMALL $C$

\begin{tabular}{|l|c|c|c|c|c|c|c|c|c|c|}
\hline$C$ & 1 & 2 & 3 & 4 & 5 & 6 & 7 & 8 & 9 & 10 \\
\hline$\rho_{\max }(C)$ & $\frac{1}{2}$ & $\frac{2}{3}$ & 1 & 1 & $\frac{5}{4}$ & $\frac{3}{2}$ & $\frac{3}{2}$ & $\frac{8}{5}$ & $\frac{9}{5}$ & 2 \\
\hline \hline$C$ & 11 & 12 & 13 & 14 & 15 & 16 & 24 & 32 & 48 & 64 \\
\hline$\rho_{\max }(C)$ & 2 & 2 & $\frac{13}{6}$ & $\frac{14}{6}$ & $\frac{5}{2}$ & $\frac{5}{2}$ & 3 & $\frac{32}{9}$ & $\frac{9}{2}$ & $\frac{64}{11}$ \\
\hline
\end{tabular}

Proof:

Case 1: Let $\frac{k(k-1)}{2} \leq C \leq \frac{(k+1)(k-1)}{2}$. If $m \leq \frac{k(k-1)}{2}$, then $\varphi(m) \leq k$ and $\rho(m) \leq \frac{k-1}{2}$. If $\frac{k(k-1)}{2}<m \leq C$, $\varphi(m)=k+1$ and $\rho(m)=\frac{m}{k+1} \leq \frac{C}{k+1}$. As $C \leq \frac{(k+1)(k-1)}{2}, \rho(m) \leq \frac{k-1}{2}$. So, we always have $\rho(m) \leq \frac{k-1}{2}$, the equality being attained for $K_{k}$ (where $m=\frac{k(k-1)}{2}$ and $\rho(m)=\frac{k-1}{2}$ ).

Case 2: Let $\frac{(k+1)(k-1)}{2} \leq C \leq \frac{(k+1) k}{2}$. If $m \leq \frac{(k+1)(k-1)}{2}$, we have seen that $\rho(m) \leq \frac{k-1}{2} \leq \frac{C}{k+1}$. If $\frac{(k+1)(k-1)}{2} \leq$ $m \leq C \leq \frac{(k+1) k}{2}, \varphi(m)=k+1$ and $\rho(m)=\frac{m}{k+1} \leq \frac{C}{k+1}$. The value $\frac{C}{k+1}$ is attained for any graph with $C$ edges and $k+1$ vertices ; such a graph can be obtained by deleting $\frac{(k+1) k}{2}-C$ edges from $K_{k+1}$.

For the sake of illustration, Table II give the values of $\rho_{\max }(C)$ for small values of $C$.

In view of Theorem III.2, we have interest to chose if possible subgraphs with a ratio equal to $\rho_{\max }(C)$. Note that according to Proposition III.3, these subgraphs do not have necessarily exactly $C$ edges and so the minimum is not necessarily attained for $W=W_{\min }$.

For example, let $C=7$. If a subgraph has 7 edges, its ratio is at most $\frac{7}{5}=1.4$. But a subgraph with 6 edges can have a ratio $\frac{6}{4}=1.5$ (and this is attained for $K_{4}$ ). Any other subgraph has a ratio at most $\frac{5}{4}$. So, in an optimal solution for the number of ADMs, we have to use as subgraphs $K_{4}$ and not subgraphs with 7 edges and 5 vertices. But in a solution minimizing the number of wavelengths, we have in contrary to use these last ones. We are now able to give counterexamples to a conjecture of [7].

Proposition III.4: The conjecture of [7] that the minimum number of ADMs, $A(C, N)$, for unidirectional rings $\vec{C}_{N}$ with uniform unitary traffic is obtained for $W=W_{\min }=\left\lceil\frac{N(N-1)}{2 C}\right\rceil$, is false.

Proof: Let $C=7$ and $N=13$. There is a decomposition of $K_{13}$ into 13 subgraphs $K_{4}$ (namely the subgraphs $B_{i}=\{i, i+1, i+4, i+6\}$ for $i=0,1, \ldots, 12$, the numbers being taken modulo 13). For this solution, $A=52$ and it is an optimal one. However, we have $W=13$ and $W_{\min }=\left\lceil\frac{78}{7}\right\rceil=12$.

Consider now a grooming with 12 subgraphs and let $W_{7}$ be the number of subgraphs with 7 edges. The $12-W_{7}$ other subgraphs have at most 6 edges each. To groom the 78 circles, we should have $7 W_{7}+6\left(12-W_{7}\right) \geq 78$, which implies $W_{7} \geq 6$. But each subgraph with 7 edges needs 5 ADMs. So, there are $5 W_{7}$ vertices to cover $7 W_{7}$ circles. To cover the remaining $78-7 W_{7}$ circles, we need by Theorem III.1 at least $\frac{4}{6}\left(78-7 W_{7}\right)$ vertices (as the ratio of any subgraph is at most $\left.\frac{6}{4}\right)$. So altogether we have a total number of vertices $A \geq 5 W_{7}+\frac{4}{6}\left(78-7 W_{7}\right) \geq 52+\left(5-\frac{28}{6}\right) W_{7}$. As $W_{7} \geq 6$, we obtain $A \geq 54$. So, conjecture of [7] is false for $C=7$ and $N=13$.

For $C=7$ we can easily build infinite families of counterexamples using known decompositions of $K_{N}$ into $K_{4}$ if $N=12 t+1$ or $12 t+4$ (see Theorem IV.4). Such a decomposition will use $W=\frac{R}{6}$ subgraphs and $A=\frac{2 R}{3}$ ADMs. On the other hand, a solution with $W_{\min }=\left\lceil\frac{R}{7}\right\rceil$ subgraphs will need around $\frac{5 R}{7}$ ADMs as all the subgraphs have exactly 7 edges and a ratio of $\frac{7}{5}$. For example if $N=85$ a solution with $W_{\min }=510$ subgraphs will need 2550 ADMs and a solution using $W=595$ subgraphs will only need 2380 ADMs, thus we can save 170 ADMs by using 85 more wavelengths. So, using $\frac{1}{6}$ more wavelength allows a saving of $\frac{1}{15}$ ADMs (in fact we can use any number of subgraphs between $\frac{R}{7}$ and $\frac{R}{6}$, increasing the number of subgraphs by 1 decreases the number of ADMs of 2 ).

Other counterexamples to the conjecture of [7] are obtained for all values of $C$ such that $\frac{k(k-1)}{2}<C<\frac{(k+1)(k-1)}{2}$, for example $C=11,16,17$. When $C=16, \rho_{\max }(16)=\frac{5}{2}$ and is attained for subgraphs $K_{6}$ which have only 15 edges.

That confirms the intuition of [17]. However, it was also conjectured in [17] that $A(16, N) \geq\left\lceil\frac{7 N(N-1)}{32}\right\rceil$. But, as we will see in Theorem IV.4, this conjecture is false, since there exists an infinite number of values of $N$ for which $A(16, N)=\frac{N(N-1)}{5}$. 


\section{UPPER BOUND AND OPTIMAL RESULTS}

Our problem looks similar to design theory. Indeed an $(N, k, 1)$-design is nothing else than a partition of the edges of $K_{N}$ into subgraphs isomorphic to $K_{k}$ called blocks in this theory. That corresponds to impose in our partitioning problem that all the subgraphs $B_{\lambda}$ are isomorphic to $K_{k}$. Note that the classical equivalent definition is : given a set of $N$ elements, find a set of blocks such that each block contains $k$ elements and each pair of elements appears in exactly one block (see the handbook [8]).

More generally, a $G$-design of order $N$ (see [8] chap. 22 or [5] or [6]) consists on partition of the edges of $K_{N}$ into subgraphs isomorphic to a given graph $G$. The interest of the existence of a $G$-design is shown by the following immediate proposition.

Proposition IV.1: If there exists a $G$-design of $\operatorname{order} N$, where $G$ is a graph with at most $C$ edges and ratio $\rho_{\max }(C)$, then $A(C, N)=\frac{N(N-1)}{2 \rho_{\max }(C)}$.

Necessary conditions IV.2-Existence of a $G$-design: If there exists a $G$-design, then

(i) $\frac{N(N-1)}{2}$ should be a multiple of $E(G)$

(ii) $N-1$ should be a multiple of the greatest common divisor of the degrees of the vertices of $G$.

Wilson [23] has shown that these necessary conditions are also sufficient for large $N$. From that, we obtain

Theorem IV.3: Given $C$, for an infinite number of values of $N, A(C, N)=\frac{N(N-1)}{2 \rho_{\max }(C)}$.

Unfortunately, the values of $N$ for which Wilson's Theorem applies are very large. However, for small values of $C$, we can use exact results of design theory (see [8] chap. 22). For example, from the existence of a $G$-design for $G=K_{3}, K_{3}+e, K_{4}-e, K_{4}, K_{5}-3 e, K_{5}-2 e, K_{5}-e, K_{5}$ and $K_{6}$, where $K_{p}-\alpha e\left(\right.$ resp. $\left.K_{p}+\alpha e\right)$ denotes the graph obtained from $K_{p}$ by deleting (resp. adding) $\alpha$ edges, we obtain

Theorem IV.4:

- $A(3, N)=\frac{N(N-1)}{2}$ when $N \equiv 1$ or $3(\bmod 6)$

- $A(4, N)=\frac{N(N-1)}{2}$ when $N \equiv 0$ or $1(\bmod 8)$

- $A(5, N)=\frac{2 N(N-1)}{5}$ when $N \equiv 0$ or $1(\bmod 10)$

- $A(6, N)=A(7, N)=\frac{N(N-1)}{3}$ when $N \equiv 1$ or $4(\bmod 12)$

- $A(8, N)=\frac{5 N(N-1)}{16}$ when $N \equiv 0$ or $1(\bmod 16)$

- $A(9, N)=\frac{5 N(N-1)}{18}$ when $N \equiv 0$ or $1(\bmod 18)$

- $A(10, N)=\frac{N(N-1)}{4}$ when $N \equiv 1$ or $5(\bmod 20)$

- $A(16, N)=\frac{N(N-1)}{5}$ when $N \equiv 1(\bmod 30)$

Therefore, as we said before, it disproves the conjecture of [17] for $A(16, N)$.

Note that, if design theory can be used, the problem is slightly different. Indeed, in design theory, one looks for a partition of the edges into isomorphic subgraphs. Such partition exist only for some values of $N$. For example, for $C=3$ and $N \not \equiv 1$ or $3(\bmod 6)$, one can think that the best solution is obtained by taking as many $K_{3}$ 's as possible, but it does not necessarily lead to an optimal solution (see [2]).

Consider for example $K_{6}$. It can be partitioned into the 4 triangles $\{0,1,2\},\{0,3,4\},\{1,3,5\},\{2,4,5\}$ plus the 3 edges $\{0,5\},\{1,4\},\{2,3\}$. So, all together we have 5 subgraphs and 18 ADMs. However, we can also partition $K_{6}$ into the $3 K_{3}$ 's $\{0,1,2\},\{1,3,4\},\{2,4,5\}$, the star $K_{1,3}\{0 \mid 3,4,5\}$, and the path $P_{4}(1,5,3,2)$. This solution use 5 subgraphs and 17 ADMs.

In [2], we determined exactly the value of $A(3, N)$, proving the following theorem.

Theorem IV.5-[2]:

(i) When $N$ is odd, $A(3, N)=\frac{N(N-1)}{2}+\epsilon$, where $\epsilon=0$ if $N \equiv 1$ or $3(\bmod 6)$, and $\epsilon=2$ if $N \equiv 5(\bmod 6)$;

(ii) When $N$ is even, $A(3, N)=\frac{N(N-1)}{2}+\left\lceil\frac{N}{4}\right\rceil+\epsilon$, where $\epsilon=1$ if $N \equiv 8(\bmod 12)$, and $\epsilon=0$ elsewhere.

The proof uses technics inspired of design theory. In the even case, the optimal solutions use a lot of $K_{3}$ 's and some $K_{1,3}$ or $P_{4}$. For example, if $n \equiv 0$ or $4(\bmod 12)$, the optimal solution consists of $\frac{N(N-1)}{6}-\frac{N}{4} K_{3}$ 's and $\frac{N}{4} K_{1,3}$. An other difference with design theory is that we can use in our decomposition a mixture of graphs with ratio $\rho_{\max }(C)$.

For example, if $C=4$, we can use either a $C_{4}$ or $K_{3}+e$ (or also a $K_{3}$ ), all these graphs having ratio $\rho_{\max }(4)=1$. It becomes easy (see [4]) to show that for $N>4, K_{N}$ can be partitioned into $C_{4}^{\prime} s$ and $K_{3}+e$, giving the result of [17] that $A(4, N)=\frac{N(N-1)}{2}$ with the minimum number of wavelengths. 
An other example is given with $C=12$, where we can use as subgraphs of the partition of $K_{N}$ either $K_{5}$ or any graph with 6 vertices and 12 edges (obtained by deleting 3 edges from $K_{6}$ ). Such a decomposition exists for example if $n \equiv 1(\bmod 4)$ in which case $A(12, N)=\frac{N(N-1)}{4}$ (see [4]).

In [3] we also determined exactly the value of $A(5, N)$.

Note that for $C=3,4,5$ there always exist solutions minimizing both the number of ADMs and the number of subgraphs (wavelengths) so conjecture of [7] is true for $C=3,4,5$.

Although the problem is asymptotically solved, for small values of $N$ (corresponding to the practical ones) we have to refine both lower and upper bounds to obtain the exact value of $A(C, N)$.

For upper bounds, we have to construct decompositions. If the graph with $\rho_{\max }(C)$ is $K_{k}$, we can use the known results of design theory, and in any case we can use the ideas of this theory in particular all the recursive constructions. This methodology is that used in [9] for $C=8$. The ideas of partitioning into classes of [7] or others appears as a particular case of this design techniques. For example, in [7] they use mainly subgraphs of the form $K_{\sqrt{C}, \sqrt{C}}$. If for $C=4$, their use is good because $K_{2,2}=C_{4}$ and $\rho_{\max }(4)=1$, in all other cases it gives result far from the optimal. For $C=16, K_{4,4}$ has a ratio 2 to be compared with $K_{6}$ which has ratio $\frac{5}{2}$. So their solution can have only at most $80 \%$ of ADMs compared to the optimal solution. Using a partition into 3 classes, we have shown

Theorem IV.6-[4]: When $C \geq R / 3, A(C, N) \leq 2 N$, except when $N=4$ and $C=2$, and when $N=7$ and $C=7$.

If exact optimal solutions might be difficult to obtain (in particular to prove the non existence of a solution), solution differing by a small constant can be easily found as there exist a lot of them. That explains why the results obtained by simulated annealing [22] are relatively good. Furthermore, as we know by the theory the structure of the optimal or quasi-optimal solutions, we can use programming by imposing constraints and looking only to particular solutions. More details will be found in forthcoming papers and the values reported in Table I where obtained in that way.

For lower bounds, if $N$ is small, we have to take into account the fact that subgraphs should have large intersections and so edges are covered many times.

Proposition IV.7: When $C \geq \frac{N(N-1)}{2}, A(C, N)=N$.

Proof: There should be at least one ADM in each vertex, so $A(C, N) \geq N$. A solution is obtained by taking a unique subgraph. In that case the traffic is carried on one wavelength (it correspond to the shaded data in [22]).

Proposition IV.8: When $\frac{N(N-1)}{4} \leq C \leq \frac{N(N-1)}{2}, A(C, N)=N+\varphi\left(\frac{N(N-1)}{2}-C\right)$.

Proof: Recall that $\varphi(m)$ is the smallest integer $k$ such that $\frac{k(k-1)}{2} \geq m$, and let $k_{0}=\varphi\left(\frac{N(N-1)}{2}-C\right)$. If each vertices belongs to at least 2 subgraphs then $A(C, N) \geq 2 N \geq N+k_{0}$. So one vertex belongs to exactly one subgraph which should contains the $N-1$ other vertices and at most $C$ edges. To cover the $\frac{N(N-1)}{2}-C$ remaining edges, we need a subgraph with at least $k_{0}$ vertices. Therefore, $A(C, N) \geq N+k_{0}$.

A solution with $N+k_{0}$ ADMs is obtained by taking two subgraphs, one with $k_{0}$ vertices covering $\frac{k_{0}\left(k_{0}-1\right)}{2}$ edges, where $\frac{k_{0}\left(k_{0}-1\right)}{2} \geq \frac{N(N-1)}{2}-C$ by definition of $k_{0}$. The second subgraphs contains all the vertices and cover the remaining edges in number less than or equal to $C$.

Applying Proposition IV.8, we obtained the results of Table I for $C=64$ and $12 \leq N \leq 16$, and for $C=48$ and $11 \leq N \leq 14$. For other values of $C$ and $N$, we have to use more sophisticated arguments (see [4]).

\section{CONCLUSION}

In this article, we have shown how the problem of traffic grooming in unidirectional WDM ring with uniform unitary traffic can be modelized as a problem of partition of the edges of a complete graph. The use of graph theory and design tools has enabled us to solve optimally the problem for practical values and infinite congruence classes of values for a given $C$. This modelization and the tools can easily extended to uniform but non unitary traffic. Indeed, if the requests satisfy $r_{i, j}=r$, it suffices to consider decomposition of the edges of the complete multipartite graph $r K_{N}$ (here again, this has been done in design theory for partition into $K_{k}$ under the name of $(N, k, r)$-design). We can also extend the ideas to the case of an arbitrary traffic, but it requires to partition general graphs and this is known to be a difficult problem in graph theory. However, our tools provide insight for finding approximate and heuristic solutions for arbitrary traffic. We can also consider networks different from the unidirectional ring, if we are first able to groups the requests into circles (that is the way used in $[9,10]$ for bidirectional rings). Finally, the tools can also be used to 
groom traffic in a slightly different context, for example, in the RNRT project PORTO our team developed with France Telecom and Alcatel, the traffic was expressed in terms of STM-1 (each one needed one wavelength) and we group them into bands or fibers, typically a fiber containing 8 bands of 4 wavelengths [18].

\section{REFERENCES}

[1] B. Beauquier, J-C. Bermond, L. Gargano, P. Hell, S. Pérennes, and U. Vaccaro. Graph problems arising from wavelength-routing in all-optical networks. In IEEE Workshop on Optics and Computer Science, Geneva, Switzerland, apr 1997.

[2] J-C. Bermond and S. Ceroi. Minimizing SONET ADMs in unidirectional WDM ring with grooming ratio 3. Networks, 41(2), February 2003.

[3] J-C. Bermond, C. Colbourn, A. Linq, and M-L. Yu. Grooming in unidirectional rings: $K_{4}-e$ designs. submitted to Discrete Applied Math.

[4] J-C. Bermond, D. Coudert, and X. Muñoz. Traffic grooming in unidirectional WDM ring networks: the all-to-all unitary case. In The 7th IFIP Working Conference on Optical Network Design \& Modelling, February 2003.

[5] J-C. Bermond, C. Huang, A. Rosa, and D. Sotteau. Decomposition of complete graphs into isomorphic subgraphs with five vertices. Ars Combinatoria, pages 211-254, 1980.

[6] J-C. Bermond and D. Sotteau. Graph decompositions and $g$-designs. In 5th British Combinatorial conference, Congressus Numerantium 15 Utilitas math. Pub., pages 53-72, Aberdeen, 1975.

[7] A. L. Chiu and E. H. Modiano. Traffic grooming algorithms for reducing electronic multiplexing costs in WDM ring networks. IEEE/OSA Journal of Lightwave Technology, 18(1):2-12, January 2000.

[8] C. Colbourn and J. Dinitz, editors. The CRC handbook of Combinatorial designs. CRC Press, 1996.

[9] C. Colbourn and A. Ling. Graph decompositions with application to wawelength add-drop multiplexing for minimizing SONET ADMs. Discrete Mathematics, 261:141-156, 2003.

[10] C. Colbourn and P-J. Wan. Minimizing drop cost for SONET/WDM networks with $\frac{1}{8}$ wavelength requirements. Networks, 37(2):107-116, March 2001.

[11] D. Coudert and H. Rivano. Lightpath assignment for multifibers WDM optical networks with wavelength translators. In IEEE Globecom, Taipei, Taiwan, November 2002.

[12] R. Dutta and N. Rouskas. A survey of virtual topology design algorithms for wavelength routed optical networks. Optical Networks, 1(1):73-89, January 2000.

[13] R. Dutta and N. Rouskas. On optimal traffic grooming in WDM rings. IEEE Journal of Selected Areas in Communications, 20(1):1-12, January 2002.

[14] R. Dutta and N. Rouskas. Traffic grooming in WDM networks: Past and future. Technical report, CSC TR-2002-08, NCSU, 2002.

[15] O. Gerstel, P. Lin, and G. Sasaki. Wavelength assignment in a WDM ring to minimize cost of embedded sONET rings. In IEEE Infocom, pages 94-101, San Francisco, California, 1998.

[16] O. Gerstel, R. Ramaswani, and G. Sasaki. Cost-effective traffic grooming in WDM rings. IEEE/ACM Transactions on Networking, 8(5):618-630, 2000.

[17] J.Q. Hu. Optimal traffic grooming for wavelength-division-multiplexing rings with all-to-all uniform traffic. OSA Journal of Optical Networks, 1(1):32-42, 2002.

[18] G. Huiban, S. Pérennes, and M. Syska. Traffic grooming in WDM networks with multi-layer switches. In IEEE ICC, New-York, April 2002.

[19] E. Modiano and P. Lin. Traffic grooming in WDM networks. IEEE Communications Magazine, 39(7):124-129, July 2001.

[20] A. Somani. Survivable traffic grooming in WDM networks. In D.K. Gautam, editor, Broad band optical fiber communications technology - BBOFCT, pages 17-45, Jalgaon, India, December 2001. Nirtali Prakashan. Invited paper.

[21] P-J. Wan, G. Calinescu, L. Liu, and O. Frieder. Grooming of arbitrary traffic in SONET/WDM BLSRs. IEEE Journal of Selected Areas in Communications, 18(10):1995-2003, October 2000.

[22] J. Wang, W. Cho, V. Vemuri, and B. Mukherjee. Improved approches for cost-effective traffic grooming in WDM ring networks: Ilp formulations and single-hop and multihop connections. IEEE/OSA Journal of Lightwave Technology, 19(11):1645-1653, November 2001.

[23] R.M. Wilson. Decomposition of complete graphs inot subgraphs isomorphic to a given graph. Congressus numerantium, 15:647-659, November 1976.

[24] X. Yuan and A. Fulay. Wavelength assignment to minimize the number of sONET ADMs in WDM rings. In IEEE ICC, New York, April 2002.

[25] X. Zhang and C. Qiao. On optimal all-to-all personalized connections and cost-effective designs in WDM rings. IEEE/ACM Transactions on Networking, 7(3):435-445, 1996.

[26] X. Zhang and C. Qiao. An effective and comprehensive approach for traffic grooming and wavelength assignment in SONET/WDM rings. IEEE/ACM Transactions on Networking, 8(5):608-617, 2000. 